\title{
THE SIMPLICITY OF THEORIES: ITS DEGREE AND FORM
}

\author{
JAMES W. McALLISTER
}

\begin{abstract}
SUMMARY. Almost all commentators acknowledge that among the grounds on which scientists perform theory-choices are criteria of simplicity. In general, simplicity is regarded either as only a logico-empirical quality of a theory, diagnostic of the theory's future predictive success, or as a purely aesthetic or otherwise extra-empirical property of it. This paper attempts to demonstrate that the simplicity-criteria applied in scientific practice include both a logicoempirical and a quasi-aesthetic criterion: to conflate these in an account of scientists' theorychoice is to court confusion.
\end{abstract}

Key words: empirical and extra-empirical evaluative criteria, parsimony, scientific theories, simplicity, theory-assessment and theory-choice.

\section{THE QUALITIES OF SCIENTIFIC THEORIES}

Among the tasks tackled by the philosophy of science is that of understanding the criteria by which scientists come to prefer a theory to its competitors.

Scientists are said to choose theories which will attain the greatest empirical success. In principle, a theory demonstrates empirical success by according with empirical evidence gathered from disparate sources over unlimited times. Scientists needing to choose between alternative theories do not have such leisure, however, and require criteria able to indicate now whether a theory is likely to show empirical success in the future. By analyzing what is meant by empirical success, or by recalling what features were displayed by past theories which scored notable successes of the desired kind, scientists draw up a list of features which appear to be diagnostic of such attainments. The features of theories which are taken as diagnostic of their future empirical success will here be termed their 'logico-empirical' properties; they are such qualities as, in W. H. Newton-Smith's formulation, observational nesting, fertility, a good track record, strong intra-theory support, and internal consistency. ${ }^{1}$ Scientific communities seek out and prefer theories which display these logico-empirical features, in the expectation that they will be likelier than other theories to succeed in empirical tests.

A scientist may portray his or her choices among theories as guided by no criteria other than logico-empirical ones: however, the philosopher who aims to account for scientists' actual preferences as these are recorded in the history of science will perceive the influence of a further set of criteria. It is shown for instance by G. Buchdahl that 'although falsifiability, con- 
firmation, and predictive power - all involving appeals to observational data, however conceptualized - are important criteria for the choice and acceptability of hypotheses, they are not sufficient' for an accurate reconstruction of the history of science. ${ }^{2}$ Accordingly, philosophers conclude that scientists apply in theory-choice not only logico-empirical criteria which are diagnostic of theories' future empirical success, but also extra-empirical criteria which do not lend themselves to this diagnostic role. Such additional criteria of theory-assessment are frequently characterized as 'aesthetic' or 'metaphysical'.3

The philosopher's reference to two canons of theory-choice, that of criteria diagnostic of empirical success and that of metaphysical or other extraempirical criteria, creates the task of deciding to which category belongs any particular criterion of which the application is observed in the history of science.

\section{SIMPLICITY AS A LOGICO-EMPIRICAL QUALITY}

Most accounts of theory-choice acknowledge that scientists will prefer a theory to its competitors if, the theories appearing equally worthy on other grounds, the claims of the former theory are simpler than those of the latter. ${ }^{4}$ The consensus that a simplicity-criterion operates in many episodes of theory-choice is not matched by agreement over the nature of this criterion, and more particularly over the category to which it is properly to be ascribed. On some accounts, the simplicity of a theory's claims is diagnostic of that theory's future empirical success, and the simplicity-criterion ought therefore to be counted among the logico-empirical evaluative criteria; other accounts suggest that the simplicity of a theory is wholly uncorrelated to its future empirical performance, and that simplicity-criteria are to be likened in this regard to many a metaphysical criterion of theory-evaluation. ${ }^{5}$ Expressions of the latter view will be discussed in the next section; first let us consider accounts of the simplicity of a theory as a criterion diagnostic of its future empirical success.

There are two common arguments to the effect that, of a pair of theories which fit current experimental data equally well, the simpler is empirically superior; ${ }^{6}$ each of these presupposes that there exist yardsticks by which the degree of simplicity of theories may be measured or at least compared. ${ }^{\text {? }}$

The first argument rests on the premises that the more informative of two hypotheses is the empirically superior, and that the simpler of a pair of theories is the more informative. E. Sober writes in justification of the first premise: 'The more informative our knowledge claims are about the individuals in our environment, the less we need to find out about the special details of any arbitrary individual before we can say what its properties are. ${ }^{8} \mathrm{~S}$. F. Barker strives to ground the second premise as follows: 
If one system is simpler than another [...] then the simpler one 'says more,' it has 'more content,' because it excludes a greater number of possible models; therefore it runs more risk of being contradicted by the evidence. A system which takes a risk yet survives deserves more credit, it earns more credibility, than does a system which survives but says less and thus has taken less risk. ${ }^{9}$

On this argument, the simpler theory is a superior predictive tool by dint of being, on its own, more informative than a more complex theory would be.

The second argument seeks to establish that simpler theories have higher likelihood of being true than more complex theories which fit the experimental findings equally well: on a Bayesian analysis, the former are therefore better supported by those data. ${ }^{10}$ The notion that simpler theories yielding the same predictions as more complex ones derive stronger support from any common body of favourable evidence has been applied on several notable occasions in scientific practice: G. C. Williams, for instance, uses a principle of parsimony to argue in evolutionary biology that the theory of group selection and biotic adaptation is less well supported by the data than is the theory of genic selection and organic adaptation. ${ }^{11}$

\section{SIMPLICITY AS AN EXTRA-EMPIRICAL OR AESTHETIC QUALITY}

The opinion that the simplicity of a theory is diagnostic of the theory's future empirical success, and thus that the simplicity-criterion belongs to the category of logico-empirical evaluative criteria, is widespread; similarly popular is however the alternative option, that of denying to the simplicity of a theory any correlation with its future empirical success. For instance, Newton-Smith writes: 'There is no reason to see greater relative simplicity $[\ldots]$ as an indicator of greater verisimilitude" in a theory. ${ }^{12}$ If this is true, the simplicity-criterion should be allocated to the extra-empirical canon of theory-evaluation. Buchdahl inclines to this view in entering 'maxims of simplicity and economy' alongside 'general metaphysical notions' in the class of extra-empirical criteria. ${ }^{13}$

What grounds suggest that simplicity-criteria cannot be diagnostic of the likely degree of future empirical success of theories? It is claimed often that the content of simplicity-criteria is subjective, and that they therefore cannot be taken as a guide to the degree to which theories possess some objective quality, such as proximity to the truth. Two arguments might be envisaged for supposing that simplicity-judgements of theories are subjective in the way this belief requires.

The first notes the indeterminacy of the expression 'the simpler of two theories'. Suppose the following: that we have need to select a theory to describe a certain physical regularity; that several theories are currently candidates for adoption, each of which purports to model the regularity by means of a polynomial function, and distinguishes itself from the other theories only in proposing a different polynomial to this end; and that all these theories accord equally well with the data now available, so we 
propose to choose 'the simplest' among them. There are, R. Harré points out, (at least) three different simplicity-criteria in terms of which we could interpret this policy:

(a) the criterion of integral numbers, upon which we would prefer a polynomial containing only integral powers to one containing fractional powers;

(b) the criterion of the lowest power, according to which we should choose the polynomial having the lowest degree;

(c) the criterion of the reduction of observables, in accordance with which we should choose the function containing the smallest number of independent variables. ${ }^{14}$

The injunction to choose the theory incorporating 'the simplest relation' offers no guidance on which of these criteria is to be applied: each of these kinds of simplicity is of arguably equal privilege. In view of this proliferation of kinds of simplicity, the decision over which of two theories is the simpler can be subjective only: judgements of simplicity, the argument concludes, cannot therefore constitute logico-empirical criteria of theorychoice. $^{15}$

The second argument aiming to demonstrate the subjectivity of simplicityjudgements hangs on an interpretation of what is actually meant by the statement that a theory is simple. Harré suggests that, at least frequently, such a statement means no more than that the theory appears pleasingly familiar to those acquainted with it:

In many cases when a theory is judged to be simple attention is not being drawn to the paucity of concepts employed in its construction or to the simplicity of its structure but to the fact that the model which it is based upon is one which either the author of the theory or preferably everyone, is quite familiar. ${ }^{16}$

Thus the billiard-ball model of gases might appear simple to a classical physicist, because of the familiarity of this model to anyone versed in Newtonian mechanics. The degree of familiarity which a community has with a theory is clearly a characteristic of the current stage in the history of the community's science, and is to this extent subjective. If indeed the judgement that a theory is simple expresses no more than its current familiarity, judgements of simplicity cannot be expected to indicate which of two theories is ceteris paribus closer to the truth.

Many of the authors who deny to the simplicity of a theory a correlation with the theory's future empirical success go on to characterize it as an aesthetic or quasi-aesthetic property of the theory. H. Margenau writes of the requirement that a theory should display a property of 'simplicity and elegance', for instance. ${ }^{17}$ The assimilation of simplicity to an aesthetic property of the theory is performed not only by observers of scientific methodology but also frequently by its practitioners. Einstein for one appears to have believed that, in the words of Y. Elkana, 'simplicity was equivalent to beauty' in theory-choice. ${ }^{18}$ On this view, the decision to adopt one theory 
in preference to another in virtue of the simplicity which they exhibit is reached on criteria of aesthetic taste. ${ }^{19}$

\section{THE CONSISTENCY OF THE TWO ACCOUNTS}

Philosophers of science are presented with the option of conceiving of simplicity-criteria as logico-empirical, and that of understanding them as extra-empirical, quasi-aesthetic criteria of theory-assessment. They appear for the most part to regard these options as exclusive alternatives; they seem unwilling to conclude that both conceptions can be accommodated in a richer account of what it is for a theory to be simple. ${ }^{20}$ Almost alone, H. Reichenbach admits that scientists apply both empirical and aesthetic criteria of simplicity, but even he circumscribes the utility of the aesthetic criterion to the choice between more and less convenient expressions of logically-equivalent theories, a conception which sorely limits the influence of an aesthetic criterion of simplicity in theory-choice. ${ }^{21}$

Philosophers' belief that these alternatives are incompatible feeds into their reconstructions of scientists' acts of theory-evaluation. For instance, D.J. Hillman portrays the scientific community as divided into two camps, one regarding simplicity as nothing but a logico-empirical criterion and the other applying it in purely aesthetic judgements:

It is likely that [some scientists] will choose the simpler theory as better supported by the evidence, even though both theories are equally compatible with the evidence in their favor. [...] Other practitioners, however, feel that the notion of simplicity cannot be helpfully characterized. Simplicity is, in their opinion, much too heavily dependent on aesthetic and pragmatic considerations to be genuinely analyzable. ${ }^{22}$

The reason for which the two accounts are believed to be mutually exclusive is, I think, that the arguments reported in section 2 seem on current interpretations to militate against the thesis that any evaluation of the simplicity of theories can be ascribed to aesthetic judgement, and that the arguments cited in section 3 are seen as weighing against the proposition that any simplicity-criteria can be a guide to empirical worth. After all, one might reason, if the degree of a theory's simplicity is diagnostic of its future empirical success, one would not want to consider judgements of its simplicity as anything other than logico-empirical evaluations; and if the appraisal of a theory's simplicity is purely subjective, one would not want to consider its outcome as in any way reflecting the theory's empirical worth.

This paper will offer an interpretation of simplicity-criteria in scientific practice which, on the contrary, allows one to hold both beliefs about the simplicity of theories, namely that $(a)$ the degree of simplicity of a theory is a logico-empirical quality of it, diagnostic of its future empirical success, and that $(b)$ evaluations of theories in the matter of their simplicity cannot be expected, in other respects, to show a systematic correlation 
with the degree of the theories' empirical success. The next section will offer no arguments for either $(a)$ or $(b)$ on its own to add to those already reported in sections 2 and 3 respectively, but will concentrate instead on demonstrating the cogency of holding both conclusions within the new account of simplicity-criteria in science.

\section{DEGREES AND FORMS OF SIMPLICITY}

Suppose that a theory's simplicity can be completely described by specifying the values of some parameters. The question to be tackled in the latter part of this section is, how many independent parameters of this kind must be consulted in the task of ranking uniquely a number of given theories according to their simplicity? By way of an approach to this problem, the question which will initially be considered in this section is, what parameters must one fix in order to give an exhaustive description of the simplicity of a given theory?

Specification of the degree of the simplicity of a theory fails on its own to constitute an exhaustive description of the simplicity of that theory. As evidence for this, consider the following possibilities. Physical theories may be simple, as the Newtonians wished them to be, in adducing the same explanatory schemas for a wide range of phenomena; ${ }^{23}$ they may be simple, as $\mathrm{E}$. Mach wished them to be, in appealing to a small number of different material entities; ${ }^{24}$ yet again, they may be simple, as Einstein wished them to be, in resting upon an economical set of independent theoretical postulates. ${ }^{25}$

Let us express the fact that theories are simple in different ways, such as these, by the statement that they exhibit different forms of simplicity. A theory will in general exhibit more than one form of simplicity; but the degree to which a theory displays one form is uncorrelated with the degree to which it possesses another. A theory may achieve a certain degree of simplicity in reducing a given range of phenomena to a common explanatory schema, attain some other degree in respect of the breadth of the ontological domain to which it appeals, and exhibit yet a third degree of simplicity in the intricacy of the postulates upon which it rests.

We should certainly say that each of these achievements is a component of the theory's simplicity, which ought to be captured by a full description of the simplicity of the theory. Thus, a complete characterization of the simplicity of a theory would consist of a specification of the degree to which the theory exhibits each of the forms of simplicity which may be envisaged in a theory. In other terms, an exhaustive description of the simplicity of a theory consists of a record of the degree to which the theory exhibits ontological parsimony, the degree to which it exhibits moderation in the appeal to independent theoretical postulates, and so on through the forms of simplicity which theories can conceivably show.

This answers the preliminary question of this section. Now let us pass 
from considering the problem of assembling a complete description of the simplicity which a theory possesses to the problem of determining the number of parameters which one must fix in order to construct a unique evaluative ranking of some theories according to their simplicity.

A specification of the simplicity which one would wish to see exhibited by theories in a certain domain of science need not, of course, be as extensive as a complete description of the simplicity actually exhibited by a theory. Whilst the latter codification must specify the degree to which the theory exhibits each of the forms of simplicity which theories may be envisaged to possess, the former codification need indicate only a limited number of forms of simplicity, viz., those forms to which one has resolved to accord particular value in theory-assessment. In fact, a canon of criteria of theoryevaluation will typically make mention - as did e.g. the canons of Mach or of Einstein - of a single form of simplicity which the creator of the canon favours.

Even if its breadth is thus reduced, a specification of the simplicity which a scientist wishes to see embodied in theories must stipulate the values of two independent parameters: the form of the simplicity that theories ought to embody, and the degree to which they are to embody simplicity of that form. This means that a canon of theory-evaluation must typically include two simplicity-related criteria, a criterion of form and one of degree of simplicity. The canon could specify, for instance, that theories of the cosmos should display simplicity in the variety of material elements to which they appeal (rather than, say, in the values of the numerical ratios which they cite), and that they should display this simplicity to the degree of appealing to four or fewer material elements (rather than to five or more). Then, given a range of theories exhibiting diverse forms of simplicity to varying degrees, the scientist equipped with a canon of theory-evaluation will select the theory of which the simplicity best accords to his or her requirements in form and in degree. (It may well be that, whatever form of simplicity they individually pick, many scientists will say that they would like to see a theory exhibit this form to the highest possible degree: but this is no less a specification of the degree to which they would like theories to possess simplicity.)

If a canon of theory-evaluation contains anything less than a specification of both the form of simplicity which theories ought to display and the degree to which they should display that form of simplicity, it will be unable in general to rank theories uniquely in the matter of their simplicity. On the one hand, a canon which contains only a criterion of form of simplicity - i.e. which limits itself to prescribing that theories ought to display ontological parsimony, say - will be unable to discriminate between theories which display this form of simplicity to different degrees. (Arguably, of course, any theory could be said to display ontological parsimony to some degree or other.) On the other hand, the criterion of degree of simplicity does not in general deliver a unique verdict in an instance of theory-choice 
if a desired form of simplicity has not also been specified. The reason for this is the following. Without a ranking of forms of simplicity having been agreed, the advocate of any theory is in general able legitimately to claim that his or her theory is the simplest of those available, on the grounds that it exhibits some form of simplicity or other to a degree greater than do its competitors; thus, under such conditions, it is in general impossible univocally to judge which among a number of alternative theories is the simplest. ${ }^{26}$

Consider for instance the task of choosing on the grounds of simplicity between the Copernican and Keplerian theories of the solar system. The Copernican theory may legitimately be considered the simpler in making reference to only circles rather than ellipses, in view of the fact that the specification of a circle requires half as many parameters as does that of an ellipse; on the other hand, the Keplerian theory is arguably the simpler in needing recourse to a smaller number of such figures to account for the trajectory of a given planet to the degree of accuracy attained by the Copernican theory. In this case, as in general, the precept to choose the simpler theory yields a determinate outcome only after a stipulation of the form of simplicity to which preference is to be attached.

While no scientist was probably ever in need to adjudicate between the simplicity of Copernicus's theory and that of Kepler's, there are many documented episodes in which the outcome of obeying the precept to choose the theory of higher degree of simplicity of a pair would have been indeterminate in the absence of a stipulation of which of the forms of simplicity exhibited by the two was to be held preferable. Here are two examples.

As G. Holton recounts, 'Einstein and Planck debated strongly in 1914 whether the simplest physics is one that regards as basic accelerated motion (as Einstein had come to believe) or unaccelerated motion (as Planck insisted). ${ }^{27}$ While this difference of opinion persisted, even if Einstein and Planck had each wished to heed the injunction of choosing the theory which they regarded as the simpler, they would still have failed to adopt the same theory. The consensus, subsequently established, that the concept of accelerated motion is the more basic ensures that Einstein's opinion is now deemed superior to that of Planck on criteria of simplicity.

In the absence of a preference for a particular form of simplicity, the choice on simplicity-criteria between Einstein's and Newton's laws of gravitation would similarly be indeterminate. As P. A. M. Dirac writes:

One of the fundamental laws of motion is the law of gravitation which, according to Newton, is represented by a very simple equation, but, according to Einstein, needs the development of an elaborate technique before its equation can even be written down. [...] From the standpoint of higher mathematics, one can give reasons in favour of the view that Einstein's law of gravitation is actually simpler than Newton's. ${ }^{28}$

A physicist minded to choose the theory of which the equations took the simplest form would be drawn to Newtonianism, while one who aimed 
at the greatest simplicity of the overall theoretical structure would find Einstein's superior. ${ }^{29}$

\section{THE NATURES OF THE TWO SIMPLICITY-CRITERIA}

The previous section suggested that, concealed in generic talk of 'preferring simpler theories', there are in fact two simplicity-criteria employed in the evaluation of scientific theories: the criterion of the degree of simplicity of a theory, and the criterion of the form of the theory's simplicity. There is now therefore no longer the problem of determining to which category of evaluative criteria belongs 'the simplicity-criterion'. Rather, there is now the problem of determining the natures of the two criteria separately: whether each of them is a logico-empirical criterion in the sense outlined in section 2 , or an extra-empirical and arguably aesthetic criterion as suggested by the arguments reported in section 3.

I am persuaded (for whatever this declaration is worth) by the arguments of the kind retraced in section 2 that, if one has on separate grounds bestowed privilege to a certain form of simplicity, and one is presented with a pair of theories each of which can be taken to exhibit that form of simplicity and which accord equally well with the currently available data, the theory exhibiting the specified form of simplicity to the greater degree is preferable qua empirically superior to the other. If this is true, the criterion of the degree of simplicity is a logico-empirical criterion of theory-evaluation in the sense enshrined in section 2 .

What of the nature of the criterion of form of simplicity? I think there are two ways by which to attempt to demonstrate that it too is a logicoempirical criterion.

One way is to find some systematic argument which establishes a correlation between a theory's possession of a certain form of simplicity and, say, its probable degree of future empirical success: it may be that some a priori reason could be discerned for believing that theories which exhibit certain forms of simplicity are ceteris paribus more likely to be true than theories which do not. Faith in this project might descend, for instance, from the assumption that the natural world itself attributes privilege to a certain form of simplicity. But the prospects of finding such an argument seem dim. While principles of statistics may suggest that a simpler theory is ceteris paribus more likely to demonstrate empirical success than a less simple theory, they give no indication of the form or forms of simplicity apt to maximize this likelihood. The logic of inference appears to have no grounds upon which to tell whether a theory which exhibits e.g. ontological parsimony is ceteris paribus more or less likely to demonstrate empirical success than a theory which exhibits moderation in the number of theoretical postulates to which it appeals.

The second way by which one might show that a theory's possession of a certain form of simplicity is correlated with greater empirical success relies 
on a meta-inductive procedure. One could cast an inductive eye over the history of science and determine whether theories demonstrating certain forms of simplicity had as a matter of contingent fact tended to be closer to the truth - as this was later revealed - than other theories. This is the procedure advocated by Newton-Smith in his survey of the 'factors [...] which can serve as fallible indicators of likely long-term observational success':

The grounds for including any particular factor will be meta-inductive. If we can locate factors that have guided scientists in making theory choices which turned out to be correct on the ultimate test, we shall have inductive grounds for operating within the constraints of these particular inductive factors. ${ }^{30}$

If such correlations were unearthed for a particular form of simplicity, one would have meta-inductive grounds for believing that theories exhibiting simplicity of that form were ceteris paribus empirically superior to other theories.

This is an empirical project in the historiography of science; to the extent to which the nature of the criterion of form of simplicity will be revealed by this approach, it is an as yet unattained item of empirical information. On the one hand, one can easily conceive of evidence being discerned that, say, theories chosen on the criterion of ontological parsimony have in history tended to be revealed closer to the truth than theories adopted on the criterion of economy of theoretical postulates. This evidence would be of the kind apt to support the judgement that the criterion of form of simplicity is a logico-empirical criterion. On the other hand, however, the historical record on the face of it shows no correlation of the required kind between particular forms of simplicity and later empirical success. If the intuition that these correlations do not exist is borne out, it would seem that judgements of the form of theories' simplicity are extra-empirical.

In summary, this article finds that an exhaustive assessment of a theory on the grounds of its simplicity requires two independent evaluations to be made. The way in which scientists choose among theories in virtue of their simplicity cannot be understood unless the difference between these two simplicity-criteria is appreciated. If the hunches explored at the end of this paper are accurate, it is justified on logico-empirical grounds to choose for adoption from among a set of theories exhibiting a certain form of simplicity the theory which exhibits that form of simplicity to the highest degree; but the prior decision of which form or forms of simplicity one should like to see embodied in theories must be taken on grounds other than logicoempirical. It is at least arguable that such choices may be made, or have commonly in the history of science been made, on criteria of aesthetic taste. ${ }^{31}$

\section{NOTES}

\footnotetext{
1 Newton-Smith (1981), pp. 226-32. A review of earlier discussions of logico-empirical criteria of theory-choice is contained in Koertge (1979), pp. 228-40.

2. Buchdahl (1970), p. 204.
} 
${ }^{3}$ That scientists commonly evaluate theories partly on metaphysical criteria which are not diagnostic of empirical success was appreciated as early as the time of logico-positivism: Frank (1957, pp. 348-60) states that scientists in theory-choice apply a metaphysical evaluative canon alongside the truth-related criteria of agreement with observation and logical consistency. ${ }^{4}$ Even Kuhn (1977, pp. 321-3), who on separate considerations is led to doubt the existence of criteria of theory-assessment of unbounded validity, adduces simplicity as a good reason for theory-choice. The philosopher's perception of the incidence of a simplicity-criterion in scientific method is generally matched by scientists' views of their own preferences. Belinfante $(1973$, p. 3) for instance prefaces a discussion of hidden-variable theories in quantum mechanics by the statement that "physicists call a theory satisfactory if (1) it agrees with the experimental facts, (2) it is logically consistent, and (3) it is simple as compared to other explanations.'

5 For general surveys of accounts of simplicity-criteria in theory-assessment see Hesse (1967), pp. 445-6, or Johnson (1982), pp. 79-83.

${ }^{6}$ Salmon (1961, pp. 275-6) discusses the kinds of link which might hold between the simplicity of a theory and its truthlikeness or empirical success.

7 A discussion of the criteria by which the degree of simplicity of a theory may be measured is offered in Hillman (1962).

${ }^{8}$ Sober (1975), p. 3.

9 Barker (1957), pp. 181-2. Barker's explanation of the value of simplicity in theory-preference would win the agreement of Popper: see e.g. his (1959), pp. 140-2.

${ }^{10}$ For the Bayesian account of simplicity see Kemeny (1953) and Rosenkrantz (1976).

11 Williams (1966), pp. 123-4. The use of simplicity-criteria in assessing competing theories in evolutionary biology is discussed in Sober (1987), pp. 212-8, and (1988), of which pp. 37-69 address simplicity directly. For a survey of the uses of simplicity-criteria in phylogenetic systematics see Johnson (1982), pp. 83-8.

12 Newton-Smith (1981), p. 231; for a similar formulation see his (1978), p. 77. The existence of any correlation between the simplicity of a theory and its truthlikeness has been denied also by Bunge (1963), pp. 96-8, and Harré (1972), p. 45.

${ }_{13}$ Buchdahl (1970), p. 206.

14 Harré (1960), pp. 138-9.

is Such an argument, to the effect that there are many conceivable kinds of simplicity, and none to which one can appeal on objective grounds in theory-evaluation, is briefly pursued by Priest (1976), pp. 436-7.

${ }^{16}$ Harre (1960), p. 143; the suggestion that the most familiar construction will be the one adjudged the simplest is made also by Priest (1976), p. 437.

17 Margenau (1950), pp. 75-101. Harré (1960, p. 147) too likens a theory's properties of 'simplicity' and 'elegance'.

18 Elkana (1982), p. 222. Similarly, Wilson (1978, p. 11) considers the simplicity of a generalization to be an important component of its elegance or beauty. Scientists conversely regard unwarranted complexity as a kind of ugliness in a theory: Shanmugadhasan (1987, p. 53) writes that in 1945 Dirac "emphasized that he did not believe his quantum electrodynamics was the right theory because it was so complicated and ugly.'

${ }^{19}$ Some further remarks on the aesthetic aspects of simplicity-criteria may be found in McAllister (1989), pp. 31-2.

${ }^{20}$ For instance, Feuer $(1957$, pp. 115-7) and Sober $(1984$, p. 238 , note 16) deny that the appraisal of a theory's simplicity can possess an aesthetic aspect as well as holding clues to the likely future empirical success of the theory.

${ }^{21}$ Reichenbach (1938), pp. 373-5.

${ }^{22}$ Hillman (1962), pp. 225-6.

${ }^{23}$ On simplicity-principles in Newton's work see Sober (1988), pp. 51-5.

${ }^{24}$ The requirement of ontological parsimony was prompted by Mach's view of science: 'Science [...] may be regarded as a minimal problem, consisting of the completest possible presentment of facts with the least possible expenditure of thought' $(1883$, p. 586 ; emphasis as 
in the original). Mach's criterion of simplicity is discussed in Ray (1987), pp. 1-50.

${ }^{25}$ Commenting upon a discrepancy of up to ten per cent between the measured value of a gravitational deviation of a light-ray and the magnitude of the effect calculated from general relativity, Einstein weighed structural simplicity against any empirical deficiency of the theory: 'This thing is not particularly important, because the main significance of the theory does not lie in the verification of little effects, but rather in the great simplification of the theoretical basis of physics as a whole' (cited in Holton 1973, p. 236). The details of Einstein's view of theory-evaluation are set out chiefly in his (1949), pp. 21-33. Further discussion of Einstein's appeal to simplicity-criteria is offered in Hesse (1974), pp. 239-55, or Elkana (1982); on this topic see also Williamson (1977).

${ }^{26}$ Lakatos (1971, p. 131, note 106; emphasis as in the original) espouses this claim too: 'Simplicity can always be defined for any pair of theories $T_{1}$ and $T_{2}$ in such a way that the simplicity of $T_{1}$ is greater than that of $T_{2}$.'

${ }^{27}$ Holton (1978), p. 299, note 8; emphasis in the original.

${ }^{28}$ Dirac (1939), p. 123. Dirac comments ibid. that holding the view that Einstein's theory is simpler than Newton's 'involves assigning a rather subtle meaning to simplicity.'

${ }^{29}$ One can imagine many other cases in which a theory which looks complex on one criterion can be interpreted as simple on another, however apparently outrageous the effect of applying this second criterion. A cartoon by J. Cartier, reproduced in Gell-Mann (1988), p. 137, depicts a lecturer stating: 'At this point we notice that this equation is beautifully simplified if we assume that space-time has 92 dimensions.' If the lecturer's audience paused to ask whether our theories should aim for the simplest equations or for the least number of dimensions attributed to space-time, they would be appealing to criteria of form of simplicity.

${ }^{30}$ Newton-Smith (1981), pp. 224-5.

${ }^{31}$ A rough version of this paper was delivered in August 1989 at a seminar at the Faculty of Philosophy, University of Leiden. I am grateful to the participants for their criticism and other comments.

\section{REFERENCES}

Barker, S. F.: 1957, Induction and Hypothesis: A Study of the Logic of Confirmation, Cornell University Press, Ithaca, N.Y.

Belinfante, F. J.: 1973, A Survey of Hidden-Variable Theories, Pergamon Press, Oxford.

Buchdahl, G.: 1970, 'History of Science and Criteria of Choice', in R. H. Stuewer (ed.), Historical and Philosophical Perspectives of Science (Minnesota Studies in the Philosophy of Science, Vol. V), University of Minnesota Press, Minneapolis, pp. 204-30.

Bunge, M.: 1963, The Myth of Simplicity: Problems of Scientific Philosophy, Prentice-Hall, Englewood Cliffs, N.J.

Dirac, P. A. M.: 1939, 'The Relation Between Mathematics and Physics', Proceedings of the Royal Society of Edinburgh 59, 122-9.

Einstein, A.: 1949, 'Autobiographical Notes', in P. A. Schilpp (ed.), Albert Einstein: PhilosopherScientist, Open Court, La Salle, Ill., pp. 1-49.

Elkana, Y.: 1982, 'The Myth of Simplicity', in G. Holton and Elkana (eds.), Albert Einstein: Historical and Cultural Perspectives, Princeton University Press, Princeton, N.J., pp. $205-51$.

Feuer, L. S.: 1957, 'The Principle of Simplicity', Philosophy of Science 24, 109-22.

Frank, P.: 1957, Philosophy of Science: The Link Between Science and Philosophy, PrenticeHall, Englewood Cliffs, N.J.

Gell-Mann, M.: 1988, 'Is the Whole Universe Composed of Superstrings?', in K. Winter (ed.), Festi-Val: Festschrift for Val Telegdi, North-Holland, Amsterdam, pp. 119-40.

Harré, R.: 1960, An Introduction to the Logic of the Sciences (second edition, 1983), Macmillan, London.

Harré, R.: 1972, The Philosophies of Science: An Introductory Survey, Oxford University Press, Oxford. 
Hesse, M. B.: 1967, 'Simplicity', in P. Edwards (ed.), The Encyclopedia of Philosophy, eight volumes, Macmillan, New York, Vol. VII, pp. 445-8.

Hesse, M. B.: 1974, The Structure of Scientific Inference, Macmillan, London.

Hillman, D. J.: 1962, 'The Measurement of Simplicity', Philosophy of Science 29, 225-52.

Holton, G.: 1973, Thematic Origins of Scientific Thought: Kepler to Einstein, Harvard University Press, Cambridge, Mass.

Holton, G.: 1978, The Scientific Imagination: Case Studies, Cambridge University Press, Cambridge.

Johnson, R.: 1982, 'Parsimony Principles in Phylogenetic Systematics: A Critical Re-appraisal', Evolutionary Theory 6, 79-90.

Kemeny, J. G.: 1953, 'The Use of Simplicity in Induction', Philosophical Review 62, 391408.

Koertge, N.: 1979, 'The Problem of Appraising Scientific Theories', in P. D. Asquith and H. E. Kyburg (eds.), Current Research in the Philosophy of Science: Proceedings of the P.S.A. Critical Research Problems Conference, Philosophy of Science Association, East Lansing, Mi., pp. 228-51.

Kuhn, T. S.: 1977, The Essential Tension: Selected Studies in Scientific Tradition and Change, University of Chicago Press, Chicago, Ill.

Lakatos, I.: 1971, 'History of Science and Its Rational Reconstruction', in R. C. Buck and R. S. Cohen (eds.), PSA 1970: Proceedings of the 1970 Biennial Meeting, Philosophy of Science Association (Boston Studies in the Philosophy of Science, Vol. VIII), Reidel, Dordrecht, pp. 91-136.

Mach, E.: 1883, The Science of Mechanics: A Critical and Historical Account of Its Development, transl. by T. J. McCormack (sixth edition, 1960), Open Court, La Salle, Ill.

Margenau, H.: 1950, The Nature of Physical Reality: A Philosophy of Modern Physics, McGrawHill, New York.

McAllister, J. W.: 1989, 'Truth and Beauty in Scientific Reason', Synthese 78, 25-51.

Newton-Smith, W. H.: 1978, 'The Underdetermination of Theory by Data', Proceedings of the Aristotelian Society, Supp. Vol. 52, pp. 71-91.

Newton-Smith, W. H.: 1981. The Rationality of Science, Routledge and Kegan Paul, London.

Popper, K. R.: 1959, The Logic of Scientific Discovery, Hutchinson, London.

Priest, G.: 1976, 'Gruesome Simplicity', Philosophy of Science 43, 432-7.

Ray, C.: 1987, The Evolution of Relativity, Adam Hilger, Bristol.

Reichenbach, H.: 1938, Experience and Prediction: An Analysis of the Foundations and the Structure of Knowledge, University of Chicago Press, Chicago, IIl.

Rosenkrantz, R. D.: 1976, 'Simplicity', in W. L. Harper and C. A. Hooker (eds.), Foundations of Probability Theory, Statistical Inference, and Statistical Theories of Science, three volumes, Reidel, Dordrecht, Vol. I, pp. 167-96.

Salmon, W. C.: 1961, "Comments on Barker's "The Role of Simplicity in Explanation" , in H. Feigl and G. Maxwell (eds.), Current Issues in the Philosophy of Science, Holt, Rinehart and Winston, New York, pp. 274-76.

Shanmugadhasan, S.: 1987, 'Dirac as Research Supervisor and Other Remembrances', in J. G. Taylor (ed.), Tributes to Paul Dirac, Adam Hilger, Bristol, pp. 48-57.

Sober, E.: 1975, Simplicity, Clarendon Press, Oxford.

Sober, E.: 1984, The Nature of Selection: Evolutionary Theory in Philosophical Focus, MIT Press, Cambridge, Mass.

Sober, E.: 1987, 'Parsimony and the Units of Selection', in N. J. Nersessian (ed.), The Process of Science: Contemporary Philosophical Approaches to Understanding Scientific Practice, Martinus Nijhoff, Dordrecht, pp. 211-9.

Sober, E.: 1988, Reconstructing the Past: Parsimony, Evolution, and Inference, MIT Press, Cambridge, Mass.

Williams, G. C.: 1966, Adaptation and Natural Selection: A Critique of Some Current Evolutionary Thought, Princeton University Press, Princeton, N.J. 
Williamson, R. B.: 1977, 'Logical Economy in Einstein's "On the Electrodynamics of Moving Bodies" ', Studies in History and Philosophy of Science 8, 49-60.

Wilson, E. O.: 1978, On Human Nature, Harvard University Press, Cambridge, Mass.

Faculty of Philosophy

University of Leiden

P.O. Box 9515

2300 RA Leiden

The Netherlands 